\title{
Comparative analysis of biological activity of probiotic preparations based on Enzymesporine
}

\author{
Alexander Gorobets*, and Denis Trubnikov \\ ${ }^{1}$ Kursk State Agricultural Academy named after I.I. Ivanov, 70, Karl Marx street, Kursk, 305021, Russia
}

\begin{abstract}
In the article the influence of using the microencapsulated probiotic preparation Enzymesporine with an enzyme in comparison with analogues that do not have an enzyme (microencapsulated and non-encapsulated) in pigs of Genesus selection is considered. The activity on intestinal digestion, growth and weight gain, and on hematological and biochemical parameters is studied. The period of life of pigs during the experiment was 48-98-148 days. In the course of the performed experiment on pigs of Genesus selection, after the usage of reliable results. The increase in the life weight and daily weight gain in the period from the $48^{\text {th }}$ to the $148^{\text {th }}$ day of life has been confirmed. That indicates the advantages of the microencapsulated probiotic preparation Enzymesporine with an enzyme over microencapsulated and non-encapsulated analogues without an enzyme. The general analysis of blood showed that the animals that received microencapsulated Enzymesporine with an enzyme. Along with that the content of erythrocytes and hemoglobin reached the upper physiological limit, which is significantly higher than that in the control groups. During the determination of biochemical parameters of blood a significant increase of the amount of total protein and albumins in experimental animals was detected up to 148 days of animals' life. A significantly high level of immunoglobulins was observed throughout the experiment in the tested animals. When comparing with the control group, that indicates a high resistance to diseases and a high immune status of pigs.
\end{abstract}

\section{Introduction}

In the industrial pig breeding of our country, the measures for searching and breeding of selective breeds that have the highest indications of productivity in combination with optimal economic efficiency have been undertaken. For this purpose, a hybrid of the Geneus selection was chosen, used in a number of modern livestock complexes, combining the parental hybrid sows F1 (a two-breed hybrid obtained by crossing a Canadian Yorkshire and Landrace) and boars of terminal lines - Canadian Duroc [1].

The main factor of productivity of pigs is the genetic potential, realization of which, in turn, is provided by balanced feeding, properly organized keeping of animals, prophylactic veterinary measures.

One of the effective approaches to aligning the physiological status, increasing the substance exchange and metabolism and, therefore, increasing the productivity is the use of probiotics.

Probiotics are live microorganisms that bring benefit to the host during their injection in optimal amounts [2]. The following basic requirements are imposed on probiotics: they must be pheno- and genotypically classified, kept alive, acid-resistant or enclosed in an acid-resistant capsule, capable of adhesion to the intestinal epithelium and colonization of the intestine, must not be pathogenic [3, 4]. Most often, probiotic preparations are used in powder or suspension form; the sorbed preparations are used in medicine for elimination of dysbacteriosis.

Some research have described that probiotics are able to produce enzymes. However, it is believed that the addition of a non-starch polysaccharide enzyme to the ration can significantly contribute to the coefficient of using nutrient substances and improving the efficiency of animal husbandry [5].

In order to become effective probiotics must be in the form and dosage that will allow them to reach the intestine and keep their activity, passing through the acidic environment of the stomach and entering the alkaline environment of the duodenum, created by bile [6]. Therefore, in recent times, microencapsulated forms of probiotics have begun to appear. Thus, one can obtain predetermined physical parameters and biological properties that can pass the acidic environment of the stomach without destruction and activate probiotics activity in the intestine.

The goal and objectives of our work were to conduct a comparative analysis of the influence of the microencapsulated probiotic preparation Enzymesporine with an enzyme in comparison with its analogue without an enzyme and the non-encapsulated form of Enzymesporine. This should be done to improve the live weight and average daily weight gains of pigs during fattening, including the hematological and biochemical parameters of the blood of these animals.

\footnotetext{
* Correspondence author: 128x160@mail.ru
} 


\section{Materials and methods}

The initial probiotic preparation Enzymesporine included the following strains of bacteria: Bacillus subtilis RNCIM B-314, Bacillus licheniformis RNCIM B-8054, Bacillus subtilis (Bacillus natto) RNCIM B12079.

In addition, the initial preparation included trypsin crystallic in an amount of 5\% with the goal of increasing the digestion ability in the intestine. After that the preparation was microencapsulated by the method described in RF patent No. 2736377 dated 16.11.2020 [7].

The output of finished microcapsules was composed $85-90 \%$. Microcapsules were represented as oval particles of gray-yellow color, $80-150 \mu \mathrm{m}$ in size. Previously conducted microbiological studies have shown that the number of viable probiotic bacteria in the prepared microencapsulated preparation was $5.5 \times 10^{9}$ cells in $1 \mathrm{~g}$. Moreover, the microcapsules themselves in the experiments in vitro, modulating gastric digestion, showed high acid resistance to the impact of hydrochloric acid [8].

The scientific and economic experiment on trial the microencapsulated preparation Enzymesporine with an enzyme executed on pigs of the Genesus selection was carried out in comparison with the microencapsulated Enzymesporine without an enzyme and with the initial non-encapsulated Enzymesporine at the pig-breeding complex "Otkrytie" LLC in the Korenevsky district of the Kursk region in Russia. The animals were selected according to the principle of analogues by live weight and gender.

The experimental animals were divided into the following groups:

1) experimental group No. 1 (introduction into the feed of microencapsulated preparation Enzymesporine with an enzyme at a dose of $3.0 \mathrm{~g}$ per day per 1 head, $\mathrm{n}=15$ );

2) experimental group No. 2 (introduction into the feed of microencapsulated preparation Enzymesporine at a dose of $3.0 \mathrm{~g}$ per day per 1 head, $\mathrm{n}=15)$;

3) experimental group No. 3 (introduction into the feed of preparation Enzymesporine at a dose of $5.0 \mathrm{~g}$ per day per 1 head, $n=15)$;

4) control group (without introduction into the feed of probiotic preparations, $n=15$ / intact).

The rations fully covered the animals' need for nutritional substances, vitamins and microelements according to zootechnical norms.

The total duration of the experiment was 100 days from the $48^{\text {th }}$ to the $148^{\text {th }}$ day. In the period of the experiment, the observations on the experimental animals were conducted. The general condition, appetite were taken into account; the absolute weight, average daily gains, and safety were determined. The control points of measurements were the $48^{\text {th }}$, the $98^{\text {th }}$ and the $148^{\text {th }}$ days.

For the studying of the hematological parameters in animals of all groups, $10 \mathrm{ml}$ of blood for laboratory analysis was taken. In the blood ESR, hematocrit, erythrocytes, leukocytes, hemoglobin were determined.
The biochemical analysis of blood included the determination of total protein, albumins, total immunoglobulins, urea, creatinine, bilirubin, ALT, AST, cholesterol, glucose, total calcium, inorganic phosphorus, total magnesium and iron.

Hematological parameters were determined with a help of a hematological analyzer Micro CC Vet 20. During the determination of biochemical parameters, an automatic biochemical analyzer Bio Chem-200 was used.

During statistical processing of the data, the mean value, the value of the standard deviation were calculated. Differences were considered significant at $\mathrm{p}<0.05$.

\section{Results and discussion}

The conducted by us studies have shown that the tested probiotics did not render a negative effect on the life and health of the experimental piglets. The safety of the pigs of the Genesus selection increased up to $100 \%$.

The general condition of the pigs in the period of the experiment was in the norm.

The results of the reaction of probiotics on animals in the form of the dynamics of daily weight gain are shown in Fig. 1 and Fig. 2.

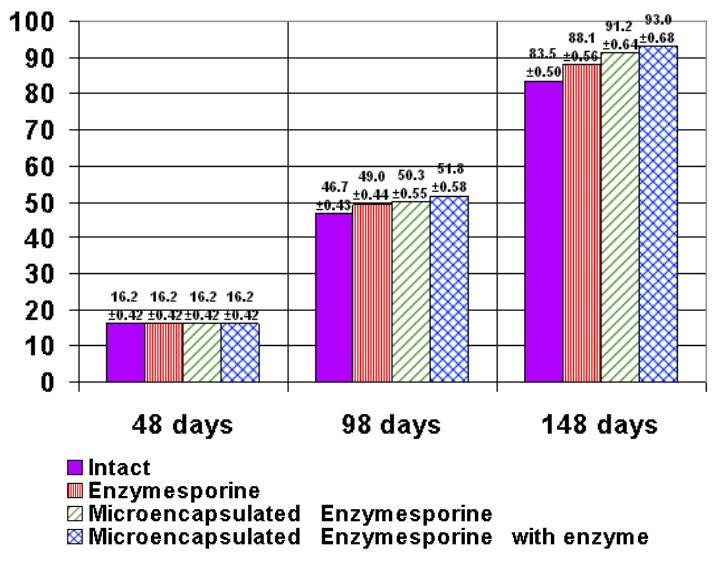

Fig. 1. Absolute weight $(\mathrm{M} \pm \mathrm{m}, \mathrm{kg})$ of pigs of the Genesus selection that participated in the scientific and economic experiment.

For evaluation of the influence of microencapsulated probiotic preparation Enzymesporine with an enzyme on the internal environment of the organism, hematological and biochemical parameters of pigs of experimental group No.1 were determined by us. They were compared to two experimental groups and the control group. The obtained results are presented in Tables 1-3. 


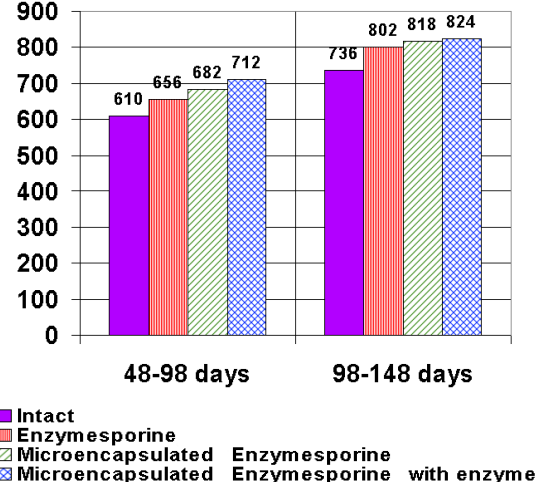

Fig. 2. Weight gain (g) of pigs of the Genesus selection that participated in the scientific and economic experiment

Table 1. Average values of hematological and biochemical parameters of pigs of Genesus selection on the $48^{\text {th }}$ day $(n=15)$

\begin{tabular}{|c|c|c|c|c|}
\hline Indications & $\begin{array}{l}\text { Experi- } \\
\text { mental } \\
\text { group } \\
\text { No.1 }\end{array}$ & $\begin{array}{c}\text { Experi- } \\
\text { mental } \\
\text { group } \\
\text { No.2 }\end{array}$ & $\begin{array}{l}\text { Experi- } \\
\text { mental } \\
\text { group } \\
\text { No.3 }\end{array}$ & $\begin{array}{l}\text { Control } \\
\text { group }\end{array}$ \\
\hline \multicolumn{5}{|c|}{ Average values of hematological parameters } \\
\hline ESR, mm/hour & $\begin{array}{l}3.0 \pm \\
0.09\end{array}$ & $\begin{array}{l}3.0 \pm \\
0.07\end{array}$ & $\begin{array}{l}3.1 \pm \\
0.09\end{array}$ & $\begin{array}{l}2.8 \pm \\
0.08\end{array}$ \\
\hline Hematocrit, \% & $\begin{array}{c}39.8 \pm \\
2.0 \\
\end{array}$ & $\begin{array}{c}39.6 \pm \\
2.3\end{array}$ & $\begin{array}{c}40.2 \pm \\
3.7 \\
\end{array}$ & $\begin{array}{c}40.0 \pm \\
3.1\end{array}$ \\
\hline $\begin{array}{l}\text { Erythrocytes, } \\
\mathrm{x} 10^{12} / 1 \\
\end{array}$ & $\begin{array}{l}6.8 \pm \\
0.34 \\
\end{array}$ & $\begin{array}{l}6.9 \pm \\
0.24 \\
\end{array}$ & $\begin{array}{l}7.0 \pm \\
0.26 \\
\end{array}$ & $\begin{array}{l}6.9 \pm \\
0.30 \\
\end{array}$ \\
\hline $\begin{array}{l}\text { Leukocytes, } \\
\times 10^{9} / 1\end{array}$ & $\begin{array}{l}9.4 \pm \\
0.21 \\
\end{array}$ & $\begin{array}{l}9.4 \pm \\
0.20 \\
\end{array}$ & $\begin{array}{l}9.5 \pm \\
0.21\end{array}$ & $\begin{array}{l}9.7 \pm \\
0.30\end{array}$ \\
\hline $\begin{array}{l}\text { Hemoglobin, } \\
\text { g/l }\end{array}$ & $\begin{array}{c}98.8 \pm \\
3.0 \\
\end{array}$ & $\begin{array}{c}98.7 \pm \\
2.9 \\
\end{array}$ & $\begin{array}{c}98.8 \pm \\
2.7 \\
\end{array}$ & $\begin{array}{c}97.9 \pm \\
3.8 \\
\end{array}$ \\
\hline \multicolumn{5}{|c|}{ Average values of biochemical parameters } \\
\hline $\begin{array}{l}\text { Total protein, } \\
\mathrm{g} / \mathrm{l}\end{array}$ & $\begin{array}{c}67.5 \pm \\
2.3 \\
\end{array}$ & $\begin{array}{c}67.7 \pm \\
2.2 \\
\end{array}$ & $\begin{array}{c}67.8 \pm \\
2.1 \\
\end{array}$ & $\begin{array}{c}68.0 \pm \\
2.1 \\
\end{array}$ \\
\hline Albumins, $\%$ & $\begin{array}{c}38.5 \pm \\
2.0 \\
\end{array}$ & $\begin{array}{c}38.6 \pm \\
2.0 \\
\end{array}$ & $\begin{array}{c}38.7 \pm \\
2.1 \\
\end{array}$ & $\begin{array}{c}38.5 \pm \\
2.1 \\
\end{array}$ \\
\hline $\begin{array}{l}\text { Immuno- } \\
\text { globulins, } \\
\mathrm{mg} / \mathrm{ml}\end{array}$ & $\begin{array}{c}11.7 \pm \\
0.47\end{array}$ & $\begin{array}{c}11.8 \pm \\
0.45\end{array}$ & $\begin{array}{c}12.1 \pm \\
0.44\end{array}$ & $\begin{array}{c}11.9 \pm \\
0.21\end{array}$ \\
\hline Urea, mmol/l & $\begin{array}{l}3.6 \pm \\
0.23 \\
\end{array}$ & $\begin{array}{l}3.7 \pm \\
0.22 \\
\end{array}$ & $\begin{array}{l}3.8 \pm \\
0.20 \\
\end{array}$ & $\begin{array}{l}3.5 \pm \\
0.11 \\
\end{array}$ \\
\hline $\begin{array}{l}\text { Creatinine, } \\
\mu \mathrm{mol} / 1\end{array}$ & $\begin{array}{c}0.11 \pm \\
0.03 \\
\end{array}$ & $\begin{array}{c}0.11 \pm \\
0.03 \\
\end{array}$ & $\begin{array}{c}0.10 \pm \\
0.04 \\
\end{array}$ & $\begin{array}{c}0.11 \pm \\
0.04 \\
\end{array}$ \\
\hline $\begin{array}{l}\text { Bilirubin, } \\
\mu \mathrm{mol} / \mathrm{l}\end{array}$ & $\begin{array}{l}1.12 \pm \\
0.12\end{array}$ & $\begin{array}{l}1.11 \pm \\
0.13\end{array}$ & $\begin{array}{c}1.09 \pm \\
0.16\end{array}$ & $\begin{array}{c}1.10 \pm \\
0.17\end{array}$ \\
\hline ALT, IU/l & $\begin{array}{c}12.0 \pm \\
0.73 \\
\end{array}$ & $\begin{array}{l}11.9 \pm \\
0.75 \\
\end{array}$ & $\begin{array}{c}11.7 \pm \\
0.80 \\
\end{array}$ & $\begin{array}{c}11.7 \pm \\
0.69 \\
\end{array}$ \\
\hline AST, IU/l & $\begin{array}{c}26.7 \pm \\
0.93\end{array}$ & $\begin{array}{c}26.5 \pm \\
0.94\end{array}$ & $\begin{array}{c}27.1 \pm \\
0.73\end{array}$ & $\begin{array}{c}26.3 \pm \\
0.74\end{array}$ \\
\hline ALP, IU/l & $\begin{array}{c}51.5 \pm \\
3.8 \\
\end{array}$ & $\begin{array}{c}51.3 \pm \\
3.7\end{array}$ & $\begin{array}{c}50.7 \pm \\
4.4\end{array}$ & $\begin{array}{c}50.9 \pm \\
4.3\end{array}$ \\
\hline $\begin{array}{l}\text { Cholesterol, } \\
\mathrm{mmol} / \mathrm{l}\end{array}$ & $\begin{array}{c}1.67 \pm \\
0.20 \\
\end{array}$ & $\begin{array}{c}1.68 \pm \\
0.23 \\
\end{array}$ & $\begin{array}{c}1.70 \pm \\
0.27 \\
\end{array}$ & $\begin{array}{c}1.71 \pm \\
0.20 \\
\end{array}$ \\
\hline $\begin{array}{l}\text { Glucose, } \\
\mathrm{mmol} / \mathrm{l}\end{array}$ & $\begin{array}{c}2.47 \pm \\
0.11\end{array}$ & $\begin{array}{c}2.48 \pm \\
0.11\end{array}$ & $\begin{array}{c}2.51 \pm \\
0.12\end{array}$ & $\begin{array}{c}2.50 \pm \\
0.10\end{array}$ \\
\hline $\begin{array}{l}\text { Total calcium, } \\
\mathrm{mmol} / \mathrm{l}\end{array}$ & $\begin{array}{c}2.44 \pm \\
0.10 \\
\end{array}$ & $\begin{array}{c}2.47 \pm \\
0.11 \\
\end{array}$ & $\begin{array}{c}2.50 \pm \\
0.10 \\
\end{array}$ & $\begin{array}{c}2.49 \pm \\
0.11 \\
\end{array}$ \\
\hline $\begin{array}{l}\text { Inorganic } \\
\text { phosphorus, } \\
\text { mmol/1 }\end{array}$ & $\begin{array}{c}1.70 \pm \\
0.09\end{array}$ & $\begin{array}{c}1.69 \pm \\
0.10\end{array}$ & $\begin{array}{c}1.68 \pm \\
0.12\end{array}$ & $\begin{array}{c}1.62 \pm \\
0.14\end{array}$ \\
\hline $\begin{array}{l}\text { Magnesium, } \\
\mathrm{mmol} / \mathrm{l}\end{array}$ & $\begin{array}{c}1.34 \pm \\
0.08 \\
\end{array}$ & $\begin{array}{c}1.31 \pm \\
0.10 \\
\end{array}$ & $\begin{array}{c}1.19 \pm \\
0.11 \\
\end{array}$ & $\begin{array}{c}1.22 \pm \\
0.15 \\
\end{array}$ \\
\hline Iron, $\mu \mathrm{mol} / 1$ & $\begin{array}{c}28.9 \pm \\
0.93 \\
\end{array}$ & $\begin{array}{c}28.6 \pm \\
0.88 \\
\end{array}$ & $\begin{array}{c}28.4 \pm \\
0.80 \\
\end{array}$ & $\begin{array}{c}28.8 \pm \\
0.90\end{array}$ \\
\hline
\end{tabular}

Table 2. Average values of hematological and biochemical parameters of pigs of Genesus selection on the $98^{\text {th }}$ day $(n=15)$

\begin{tabular}{|c|c|c|c|c|}
\hline Indications & $\begin{array}{c}\text { Experi- } \\
\text { mental } \\
\text { group } \\
\text { No.1 }\end{array}$ & $\begin{array}{l}\text { Experi- } \\
\text { mental } \\
\text { group } \\
\text { No.2 }\end{array}$ & $\begin{array}{c}\text { Experi- } \\
\text { mental } \\
\text { group } \\
\text { No.3 }\end{array}$ & $\begin{array}{c}\text { Control } \\
\text { group }\end{array}$ \\
\hline \multicolumn{5}{|c|}{ Average values of hematological parameters } \\
\hline $\begin{array}{l}\text { ESR, } \\
\mathrm{mm} / \text { hour }\end{array}$ & $\begin{array}{l}2.5 \pm \\
0.08\end{array}$ & $\begin{array}{l}2.7 \pm \\
0.09\end{array}$ & $\begin{array}{l}2.9 \pm \\
0.07\end{array}$ & $\begin{array}{l}2.7 \pm \\
0.07\end{array}$ \\
\hline $\begin{array}{l}\text { Hematocrit, } \\
\%\end{array}$ & $\begin{array}{c}41.0 \pm \\
3.1\end{array}$ & $\begin{array}{c}41.0 \pm \\
2.7\end{array}$ & $\begin{array}{c}40.0 \pm \\
2.6\end{array}$ & $\begin{array}{c}40.0 \pm \\
3.0\end{array}$ \\
\hline $\begin{array}{l}\text { Erythrocytes, } \\
\times 10^{12} / 1\end{array}$ & $\begin{array}{l}6.9 \pm \\
0.24\end{array}$ & $\begin{array}{l}6.9 \pm \\
0.22\end{array}$ & $\begin{array}{l}6.9 \pm \\
0.30\end{array}$ & $\begin{array}{l}7.0 \pm \\
0.20\end{array}$ \\
\hline $\begin{array}{l}\text { Leukocytes, } \\
\text { x10 } / 1\end{array}$ & $\begin{array}{l}9.8 \pm \\
0.18\end{array}$ & $\begin{array}{l}9.7 \pm \\
0.20\end{array}$ & $\begin{array}{l}9.5 \pm \\
0.24\end{array}$ & $\begin{array}{l}9.3 \pm \\
0.20\end{array}$ \\
\hline $\begin{array}{l}\text { Hemoglobin, } \\
\text { g/l }\end{array}$ & $\begin{array}{c}99.5 \pm \\
3.8\end{array}$ & $\begin{array}{c}98.9 \pm \\
4.0\end{array}$ & $\begin{array}{c}98.4 \pm \\
4.2\end{array}$ & $\begin{array}{c}99.0 \pm \\
3.6\end{array}$ \\
\hline \multicolumn{5}{|c|}{ Average values of biochemical parameters } \\
\hline $\begin{array}{l}\text { Total } \\
\text { protein, g/l }\end{array}$ & $\begin{array}{c}68.9 \pm \\
2.0\end{array}$ & $\begin{array}{c}68.7 \pm \\
2.7\end{array}$ & $\begin{array}{c}68.4 \pm \\
2.3\end{array}$ & $\begin{array}{c}68.1 \pm \\
3.4\end{array}$ \\
\hline Albumins, $\%$ & $\begin{array}{c}39.4 \pm \\
2.3\end{array}$ & $\begin{array}{c}39.3 \pm \\
2.1\end{array}$ & $\begin{array}{c}39.0 \pm \\
2.7\end{array}$ & $\begin{array}{c}38.8 \pm \\
2.3\end{array}$ \\
\hline $\begin{array}{l}\text { Immuno- } \\
\text { globulins, } \\
\mathrm{mg} / \mathrm{ml}\end{array}$ & $\begin{array}{l}12.0 \pm \\
0.37\end{array}$ & $\begin{array}{c}12.2 \pm \\
0.40\end{array}$ & $\begin{array}{c}12.3 \pm \\
0.38\end{array}$ & $\begin{array}{c}11.7 \pm \\
0.44\end{array}$ \\
\hline Urea, mmol/l & $\begin{array}{l}3.8 \pm \\
0.20\end{array}$ & $\begin{array}{l}3.9 \pm \\
0.19\end{array}$ & $\begin{array}{l}3.7 \pm \\
0.24\end{array}$ & $\begin{array}{l}3.7 \pm \\
0.24\end{array}$ \\
\hline $\begin{array}{l}\text { Creatinine, } \\
\mu \mathrm{mol} / \mathrm{l}\end{array}$ & $\begin{array}{c}0.10 \pm \\
0.02\end{array}$ & $\begin{array}{c}0.12 \pm \\
0.04\end{array}$ & $\begin{array}{c}0.12 \pm \\
0.05\end{array}$ & $\begin{array}{c}0.13 \pm \\
0.02\end{array}$ \\
\hline $\begin{array}{l}\text { Bilirubin, } \\
\mu \mathrm{mol} / 1\end{array}$ & $\begin{array}{c}1.23 \pm \\
0.17\end{array}$ & $\begin{array}{c}1.21 \pm \\
0.20\end{array}$ & $\begin{array}{c}1.18 \pm \\
0.19\end{array}$ & $\begin{array}{c}1.15 \pm \\
0.18\end{array}$ \\
\hline ALT, IU/1 & $\begin{array}{c}12.9 \pm \\
0.67\end{array}$ & $\begin{array}{c}12.7 \pm \\
0.85\end{array}$ & $\begin{array}{c}12.5 \pm \\
0.91\end{array}$ & $\begin{array}{c}11.6 \pm \\
0.73\end{array}$ \\
\hline AST, IU/l & $\begin{array}{c}27.4 \pm \\
0.88\end{array}$ & $\begin{array}{c}28.1 \pm \\
0.93\end{array}$ & $\begin{array}{c}28.8 \pm \\
0.50\end{array}$ & $\begin{array}{c}27.5 \pm \\
0.83\end{array}$ \\
\hline ALP, IU/l & $\begin{array}{c}50.3 \pm \\
4.7\end{array}$ & $\begin{array}{c}50.8 \pm \\
5.0\end{array}$ & $\begin{array}{l}51.8 \pm \\
3.3\end{array}$ & $\begin{array}{c}51.7 \pm \\
3.7\end{array}$ \\
\hline $\begin{array}{l}\text { Cholesterol, } \\
\mathrm{mmol} / \mathrm{l}\end{array}$ & $\begin{array}{l}1.71 \pm \\
0.24\end{array}$ & $\begin{array}{c}1.70 \pm \\
0.21\end{array}$ & $\begin{array}{c}1.69 \pm \\
0.20\end{array}$ & $\begin{array}{c}1.66 \pm \\
0.17\end{array}$ \\
\hline $\begin{array}{l}\text { Glucose, } \\
\mathrm{mmol} / \mathrm{l}\end{array}$ & $\begin{array}{c}2.68 \pm \\
0.15\end{array}$ & $\begin{array}{c}2.65 \pm \\
0.12\end{array}$ & $\begin{array}{c}2.61 \pm \\
0.15\end{array}$ & $\begin{array}{c}2.53 \pm \\
0.09\end{array}$ \\
\hline $\begin{array}{l}\text { Total } \\
\text { calcium, } \\
\mathrm{mmol} / \mathrm{l}\end{array}$ & $\begin{array}{c}2.71 \pm \\
0.12\end{array}$ & $\begin{array}{l}2.64 \pm \\
0.15\end{array}$ & $\begin{array}{c}2.58 \pm \\
0.12\end{array}$ & $\begin{array}{c}2.47 \pm \\
0.17\end{array}$ \\
\hline $\begin{array}{l}\text { Inorganic } \\
\text { phosphorus, } \\
\text { mmol/l }\end{array}$ & $\begin{array}{c}1.72 \pm \\
0.14\end{array}$ & $\begin{array}{c}1.71 \pm \\
0.12\end{array}$ & $\begin{array}{c}1.68 \pm \\
0.14\end{array}$ & $\begin{array}{c}1.71 \pm \\
0.10\end{array}$ \\
\hline $\begin{array}{l}\text { Magnesium, } \\
\mathrm{mmol} / \mathrm{l}\end{array}$ & $\begin{array}{c}1.30 \pm \\
0.10\end{array}$ & $\begin{array}{c}1.29 \pm \\
0.13\end{array}$ & $\begin{array}{c}1.28 \pm \\
0.15\end{array}$ & $\begin{array}{c}1.30 \pm \\
0.16\end{array}$ \\
\hline Iron, $\mu \mathrm{mol} / \mathrm{l}$ & $\begin{array}{c}29.7 \pm \\
0.88\end{array}$ & $\begin{array}{c}29.5 \pm \\
0.82\end{array}$ & $\begin{array}{c}28.9 \pm \\
0.74\end{array}$ & $\begin{array}{c}29.3 \pm \\
0.76\end{array}$ \\
\hline
\end{tabular}

In the course of the conducted studies, it has been found that the hematological parameters in pigs of all groups were within the limits of physiological norm. The erythrocyte sedimentation rate was $2.5 \pm 0.08-3.1 \pm 0.09$ $\mathrm{mm} /$ hour. The hematocrit value fluctuated in the range of $39.8 \pm 2.0-41.9 \pm 2.5$. Herewith, with increasing the age of the animals, it enlarged.

The content of erythrocytes and hemoglobin in pigs of all groups during setting up on the experiment was at a minimum level. However, in animals that received Enzymesporine (in the microencapsulated and nonencapsulated form without an enzyme), by the end of the 
experiment their content increased. At the same time, in pigs that received a microencapsulated probiotic with an enzyme (experiment), this increase was significant $(\mathrm{p}<0.05)$. In pigs from experimental groups No. 2 and No. 3 and the control group, changes in the content of erythrocytes and hemoglobin were statistically insignificant.

Table 3. Average values of hematological and biochemical parameters of pigs of Genesus selection on the $148^{\text {th }}$ day $(\mathrm{n}=15)$

\begin{tabular}{|c|c|c|c|c|}
\hline Indications & $\begin{array}{c}\text { Experi- } \\
\text { mental } \\
\text { group } \\
\text { No.1 } \\
\end{array}$ & $\begin{array}{c}\text { Experi- } \\
\text { mental } \\
\text { group } \\
\text { No. } 2 \\
\end{array}$ & $\begin{array}{c}\text { Experi- } \\
\text { mental } \\
\text { group } \\
\text { No.3 } \\
\end{array}$ & $\begin{array}{c}\text { Control } \\
\text { group }\end{array}$ \\
\hline \multicolumn{5}{|c|}{ Average values of hematological parameters } \\
\hline $\begin{array}{l}\text { ESR, } \\
\mathrm{mm} / \text { hour }\end{array}$ & $\begin{array}{l}2.8 \pm \\
0.07\end{array}$ & $\begin{array}{l}2.9 \pm \\
0.08\end{array}$ & $\begin{array}{l}3.1 \pm \\
0.09\end{array}$ & $\begin{array}{l}3.0 \pm \\
0.08\end{array}$ \\
\hline $\begin{array}{l}\text { Hematocrit, } \\
\%\end{array}$ & $\begin{array}{l}41.9 \pm \\
2.5\end{array}$ & $\begin{array}{l}41.5 \pm \\
2.6\end{array}$ & $\begin{array}{c}41.0 \pm \\
2.7\end{array}$ & $\begin{array}{c}40.4 \pm \\
2.8\end{array}$ \\
\hline $\begin{array}{l}\text { Erythrocytes, } \\
\times 10^{12} / 1\end{array}$ & $\begin{array}{l}7.4 \pm \\
0.10\end{array}$ & $\begin{array}{l}7.3 \pm \\
0.14\end{array}$ & $\begin{array}{l}7.3 \pm \\
0.12\end{array}$ & $\begin{array}{l}6.9 \pm \\
0.11\end{array}$ \\
\hline $\begin{array}{l}\text { Leukocytes, } \\
\times 10^{9} / 1\end{array}$ & $\begin{array}{l}9.9 \pm \\
0.20\end{array}$ & $\begin{array}{l}9.7 \pm \\
0.21\end{array}$ & $\begin{array}{l}9.6 \pm \\
0.17\end{array}$ & $\begin{array}{l}9.8 \pm \\
0.24\end{array}$ \\
\hline $\begin{array}{l}\text { Hemoglobin, } \\
\text { g/l }\end{array}$ & $\begin{array}{c}108.5 \pm \\
3.0 \\
\end{array}$ & $\begin{array}{c}106.4 \pm \\
3.1 \\
\end{array}$ & $\begin{array}{c}104.5 \pm \\
3.2 \\
\end{array}$ & $\begin{array}{c}99.4 \pm \\
2.7 \\
\end{array}$ \\
\hline \multicolumn{5}{|c|}{ Average values of biochemical parameters } \\
\hline $\begin{array}{l}\text { Total } \\
\text { protein, } g / 1\end{array}$ & $\begin{array}{c}78.7 \pm \\
2.0\end{array}$ & $\begin{array}{c}74.6 \pm \\
1.9\end{array}$ & $\begin{array}{c}69.8 \pm \\
2.1\end{array}$ & $\begin{array}{c}68.7 \pm \\
1.5\end{array}$ \\
\hline Albumins, $\%$ & $\begin{array}{c}44.5 \pm \\
2.0 \\
\end{array}$ & $\begin{array}{c}43.7 \pm \\
1.9 \\
\end{array}$ & $\begin{array}{c}43.0 \pm \\
1.9 \\
\end{array}$ & $\begin{array}{c}39.1 \pm \\
1.8 \\
\end{array}$ \\
\hline $\begin{array}{l}\text { Immuno- } \\
\text { globulins, } \\
\mathrm{mg} / \mathrm{ml}\end{array}$ & $\begin{array}{c}19.9 \pm \\
0.26\end{array}$ & $\begin{array}{c}17.8 \pm \\
0.25\end{array}$ & $\begin{array}{c}15.5 \pm \\
0.20\end{array}$ & $\begin{array}{l}12.4 \pm \\
0.23\end{array}$ \\
\hline Urea, $\mathrm{mmol} / \mathrm{l}$ & $\begin{array}{l}3.7 \pm \\
0.17\end{array}$ & $\begin{array}{l}3.8 \pm \\
0.19\end{array}$ & $\begin{array}{l}3.9 \pm \\
0.26\end{array}$ & $\begin{array}{l}4.1 \pm \\
0.27\end{array}$ \\
\hline $\begin{array}{l}\text { Creatinine, } \\
\mu \mathrm{mol} / 1\end{array}$ & $\begin{array}{c}0.11 \pm \\
0.03\end{array}$ & $\begin{array}{c}0.11 \pm \\
0.03\end{array}$ & $\begin{array}{c}0.10 \pm \\
0.03\end{array}$ & $\begin{array}{c}0.10 \pm \\
0.03\end{array}$ \\
\hline $\begin{array}{l}\text { Bilirubin, } \\
\mu \mathrm{mol} / 1\end{array}$ & $\begin{array}{c}1.27 \pm \\
0.10 \\
\end{array}$ & $\begin{array}{c}1.21 \pm \\
0.13 \\
\end{array}$ & $\begin{array}{c}1.10 \pm \\
0.22 \\
\end{array}$ & $\begin{array}{c}1.20 \pm \\
0.15 \\
\end{array}$ \\
\hline ALT, IU/1 & $\begin{array}{c}14.7 \pm \\
0.79\end{array}$ & $\begin{array}{c}15.0 \pm \\
0.72\end{array}$ & $\begin{array}{c}15.4 \pm \\
0.53\end{array}$ & $\begin{array}{c}12.0 \pm \\
0.68\end{array}$ \\
\hline AST, IU/1 & $\begin{array}{c}28.8 \pm \\
0.81 \\
\end{array}$ & $\begin{array}{c}29.6 \pm \\
0.63\end{array}$ & $\begin{array}{c}29.9 \pm \\
0.46\end{array}$ & $\begin{array}{c}26.5 \pm \\
0.60\end{array}$ \\
\hline ALP, IU/1 & $\begin{array}{c}52.2 \pm \\
4.9\end{array}$ & $\begin{array}{c}52.1 \pm \\
4.2\end{array}$ & $\begin{array}{c}52.0 \pm \\
3.0\end{array}$ & $\begin{array}{c}51.8 \pm \\
3.5\end{array}$ \\
\hline $\begin{array}{l}\text { Cholesterol, } \\
\mathrm{mmol} / \mathrm{l}\end{array}$ & $\begin{array}{l}1.86 \pm \\
0.24\end{array}$ & $\begin{array}{c}1.84 \pm \\
0.26\end{array}$ & $\begin{array}{c}1.80 \pm \\
0.30\end{array}$ & $\begin{array}{c}1.68 \pm \\
0.31\end{array}$ \\
\hline $\begin{array}{l}\text { Glucose, } \\
\mathrm{mmol} / \mathrm{l}\end{array}$ & $\begin{array}{c}3.11 \pm \\
0.17\end{array}$ & $\begin{array}{c}3.07 \pm \\
0.13\end{array}$ & $\begin{array}{c}3.04 \pm \\
0.19\end{array}$ & $\begin{array}{c}2.60 \pm \\
0.11\end{array}$ \\
\hline $\begin{array}{l}\text { Total } \\
\text { calcium, } \\
\mathrm{mmol} / \mathrm{l}\end{array}$ & $\begin{array}{c}3.14 \pm \\
0.11\end{array}$ & $\begin{array}{c}3.07 \pm \\
0.15\end{array}$ & $\begin{array}{c}2.89 \pm \\
0.16\end{array}$ & $\begin{array}{c}2.54 \pm \\
0.10\end{array}$ \\
\hline $\begin{array}{l}\text { Inorganic } \\
\text { phosphorus, } \\
\mathrm{mmol} / \mathrm{l}\end{array}$ & $\begin{array}{l}1.50 \pm \\
0.09\end{array}$ & $\begin{array}{c}1.49 \pm \\
0.11\end{array}$ & $\begin{array}{c}1.48 \pm \\
0.14\end{array}$ & $\begin{array}{c}1.88 \pm \\
0.12\end{array}$ \\
\hline $\begin{array}{l}\text { Magnesium, } \\
\mathrm{mmol} / \mathrm{l}\end{array}$ & $\begin{array}{c}1.11 \pm \\
0.10\end{array}$ & $\begin{array}{c}1.15 \pm \\
0.11\end{array}$ & $\begin{array}{c}1.27 \pm \\
0.12\end{array}$ & $\begin{array}{c}1.39 \pm \\
0.09\end{array}$ \\
\hline Iron, $\mu \mathrm{mol} / \mathrm{l}$ & $\begin{array}{c}28.8 \pm \\
0.90\end{array}$ & $\begin{array}{c}29.8 \pm \\
0.88\end{array}$ & $\begin{array}{c}31.8 \pm \\
0.91\end{array}$ & $\begin{array}{c}32.0 \pm \\
0.77\end{array}$ \\
\hline
\end{tabular}

In the content of leukocytes, significant differences have not been revealed.

When studying the biochemical parameters of blood, reflecting the level of metabolic processes occurring in the organism of animals in the experimental groups, we found that some indications had a general tendency to increase. Others decreased, and a part of the indications changed in unreliable limits.

The group of indications demonstrating a noticeable increase of values in our experiment was related total protein, and albumins. The content of these components in the blood of pigs that received microencapsulated Enzymesporine with an enzyme gradually increased with the age of the animals, reaching a maximum value at the $148^{\text {th }}$ day of age. In pigs of the control group, these blood parameters also increased by the end of the experiment. However, this increase had not significant character $(p>0.05)$. In pigs of the 3 rd experimental group and control group, the content of total protein and albumin, relatively to the animals of experimental groups No. 1 and No. 2, changed insignificantly.

The pigs that received a microencapsulated probiotic with an enzyme, had in their blood a significantly higher $(p<0.05)$ content of total immunoglobulins in comparison with the animals of the second, the third experimental and control groups. This points out that the microencapsulated probiotic, in combination with an enzyme, increases the defensive status of the organism.

In the blood of pigs of experimental group No. 1, an increase in the level of glucose was noted. An increase of glucose in blood in pigs that received microencapsulated Enzymesporine with an enzyme indicates the intensity of metabolic processes in the organism. Along with this, the value of this indication was somewhat reduced in comparison with the data of the second and the third experimental groups. In pigs of the control group in the glucose content, significant changes were not found.

Analysis of the quantitative content of mineral elements in the blood of various groups of animals revealed significant changes in the quantitative content of total calcium, inorganic phosphorus and magnesium. If the level of calcium significantly increased in pigs that were treated with a microencapsulated probiotic with an enzyme, the content of phosphorus and magnesium, on the contrary, significantly decreased.

The determination of ALT and AST showed that for pigs, which were treated with microencapsulated Enzymesporine, their enzymatic activity increased by the end of the experiment and was significantly higher on the 148th day than that in control animals. However, this indication did not leave over limits of the physiological norm. In the pigs of the experimental group, which were fed with microencapsulated Enzymesporine with an enzyme, significant differences with the control animals were not indicated.

In animals that were included in the control group (not received probiotic), in the content of biochemical blood parameters significant changes were not determined. Thus, the level of urea, creatinine, bilirubin, cholesterol, alkaline phosphatase in pigs of experimental groups No. 2 and No. 3 was within the physiological limits and did not have significant differences from animals of the control group.

Thus, in the determination of the general hematological and biochemical parameters, the animals 
that received a microencapsulated probiotic preparation with an enzyme had a high content - at the level of the upper physiological limit - of erythrocytes, hemoglobin, total protein, albumin, glucose, total calcium, which indicates the intensity of metabolic processes. Herewith, an increased level of immunoglobulins, obviously, testifies a high level of immunity and a higher resistance of the animals' organism.

\section{Conclusions}

Microencapsulated probiotic preparation with an enzyme exerts the largest influence on growths and weight gains of pigs of Genesus selection in comparison with the microencapsulated analogue without an enzyme, nonencapsulated analogue, and control.

When determining the hematological and biochemical parameters of blood, the animals that received the microencapsulated probiotic preparation Enzymesporine with an enzyme, had a high content (at the level of the upper physiological limit) of erythrocytes, hemoglobin, total protein, albumines, glucose, and total calcium. That indicates the intensity of metabolic processes. An increased level of immunoglobulins, obviously, testifies a high level of immunity and a higher resistance of the animals' organism. Herewith, the preparation extended a positive influence on average daily gains and absolute live weight of pigs during the fattening period.

The influence of this preparation on the degree of attaining the immunobiological status of the organism and functions of the gastrointestinal tract of pigs was studied.

Based on this, we can conclude that the microencapsulated probiotic preparation Enzymesporine with an enzyme is effective for improving the digestive function of the intestine, render an indirect effect on the growth and development of animals. In addition, it is recommended for using in the framework of large pig breeding complexes that use Genesus selection.

Thus, the microencapsulated probiotic Enzymesporine with an enzyme does itself justice. Because of that, the preparation is recommended for introduction into industrial pig breeding.

\section{Acknowledgements}

The acknowledgements are dedicated to Kharchenko Ekaterina Vladimirovna and Sein Oleg Borisovich from Kursk State Agricultural Academy, scientific and research laboratories, who provided for the experimental part of the research.

This research was funded by RFBR in the framework of scientific project No. 19-316-90011.

\section{References}

1. V. T. Chistyakov, Vestnik of Voronezh state agricultural university 4 (59), 71-78, (2018)

2. C. Hill, F. Guarner, G. Reid, G.R. Gibson, D. J. Merenstein, B. Pot, et al. Nat Rev Gastroenterol Hepatol. 11(8), 506-514, (2014)
3. E.A. Kornienko, Children's infections 6(3), 63-68, (2007)

4. M.A. Sheveleva, G.A. Ravenskaya, Antibiotiki $i$ Khimioterapiya 54(3-4), 61-68

5. Y. Ruokun, P. Yanni, L. Xingyao et al. Hindawi: Journal of Chemistry 2020, 9140281, (2020)

6. I.L. Khalif, A.O. Golovenko, I.I. Dikstein, O.V. Golovenko Experimental \& clinical gastroenterology 3, 3-10, (2013)

7. A.S. Belous, D.V. Trubnikov, E.V. Trublikova, A.Y. Gorobets, M.I. Kartashov, Parent RF, No. 2736377 (2020)

8. D.V. Trubnikov, A.Y. Gorobets, E.V. Trubnikova et al. IOP Conf. Ser.: Earth Environ. Sci. 548, 082089 ,(2020) 